\title{
Caracterização física, química e sensorial de sobremesas à base de soja, elaboradas com mucilagem de chia
}

\author{
Physical, chemical and sensory characterization of soy-based desserts made with chia mucilage
}

\author{
Jordana Corralo Spada ${ }^{I}$ Melina Dick ${ }^{\text {II }}$ Carlos Henrique Pagno ${ }^{\text {II }}$ Aline Campos Vieira ${ }^{\text {II }}$ \\ Anahí Bernstein ${ }^{\mathrm{II}}$ Chaline Caren Coghetto ${ }^{\mathrm{II}}$ Ligia Damasceno Ferreira Marczak ${ }^{\mathrm{I}}$ \\ Isabel Cristina Tessaro ${ }^{\mathrm{I}}$ Nilo Sérgio Medeiros Cardozo ${ }^{\mathrm{I}}$ Simone Hickmann Flôres ${ }^{\mathrm{I}{ }^{*}}$
}

\section{RESUMO}

No desenvolvimento de produtos, é de suma importância o conhecimento da influência das modificações da formulação nas propriedades sensoriais, físicas e químicas destes. O objetivo deste trabalho foi caracterizar sobremesas à base de soja com e sem adição de mucilagem de chia, utilizada como espessante alimentar. As formulações foram caracterizadas quanto as suas propriedades reológicas, ${ }^{\circ} \mathrm{Brix}, \mathrm{pH}$ e cor. O perfil sensorial foi determinado por Análise Descritiva Quantitativa (ADQ), utilizando-se uma equipe de 10 julgadores treinados. Os resultados mostraram que as amostras não diferiram quanto aos valores de $\mathrm{pH}$, ${ }^{\circ}$ Brix e coordenada cromática $a^{*}$. Quanto aos outros parâmetros de cor e reológicos, foram detectadas diferenças significativas entre as amostras, o que mostra a influência do tipo de espessante nestas características. Os resultados da $A D Q$ mostraram que as amostras não apresentaram diferenças estatísticas quanto aos atributos, cor rosa, sabor de goiaba, sabor de soja e cremosidade, porém diferiram quanto à consistência. Com isso, conclui-se que a adição da mucilagem de chia pode ser feita sem causar grandes modificações ao produto.

Palavras-chave: análise descritiva quantitativa, sobremesa, chia, soja, reologia.

\section{ABSTRACT}

In product development is important to study the influence of ingredients in the physical, chemical and sensory properties of the product. The aim of this study was to characterize soy-based desserts with and without addition of chia mucilage, used as food thickener. The formulations were characterized for their rheological properties, ${ }^{\circ} \mathrm{Brix}, \mathrm{pH}$ and color. The sensory profile was determined by Quantitative Descriptive Analysis (QDA) using 10 trained judges. The results regarding to $\mathrm{pH},{ }^{\circ} \mathrm{Brix}$ and color coordinate $a^{*}$ did not differ. Unlike these parameters, the other chromaticity coordinates and the rheological properties presented significant statistical differences between the samples.
The QDA results showed that the samples did not differ as to the attributes, color, pink guava flavor, creaminess and flavor of soy, but differed in consistency. Thus, it is concluded that the addition of mucilage chia can be made without causing major changes to the product.

Key words: quantitative descriptive analysis, dessert, chia, soy, rheology.

\section{INTRODUÇÃO}

Estima-se que $28 \%$ da população brasileira e aproximadamente $75 \%$ da população mundial tenham seu nível de lactase reduzido, o que pode levar à intolerância à lactose e dificuldade de digerir produtos lácteos (ALM, 2002). Assim, o desenvolvimento de alimentos destituídos de lactose tornou-se um nicho importante para as indústrias de alimentos.

Um dos mais importantes substitutos do leite, assim como produtos de origem animal, é a soja, visto que esta, assim como seus derivados, apresentam boas características nutricionais. Essa leguminosa é considerada um alimento funcional, devido ao seu alto teor de proteína, composição de gordura (rica em ácidos graxos insaturados), presença de isoflavonas e apropriado perfil de minerais essenciais (FRIEDMAN \& BRANDON, 2001).

Nos últimos anos, a semente de chia (Salvia hispanica L.), nativa da região do México, tornouse cada vez mais importante para a saúde e nutrição

\footnotetext{
'Departamento de Engenharia Química, Universidade Federal do Rio Grande do Sul (UFRGS), Porto Alegre, RS, Brasil.

IIDepartamento de Tecnologia dos Alimentos, Instituto de Ciência e Tecnologia de Alimentos, 91540-000, Porto Alegre, RS, Brasil. E- mail: simone.flores@ufgrs.br.*Autor para correspondência.
} 
humana, devido ao seu alto teor de ácidos graxos essenciais, fibra alimentar e proteínas (PEIRETTI \& GAI, 2009). Quando mergulhada em água, a semente forma um gel transparente mucilaginoso, composto essencialmente de fibras solúveis. As propriedades mucilaginosas da goma formada possuem qualidades que permitem sua aplicação em diversos produtos na indústria de alimentos (LIN et al., 1994), podendo assim melhorar características organolépticas, como a textura e o valor nutricional dos produtos elaborados.

De acordo com MUÑOZ et al. (2012), a mucilagem da chia é um novo ingrediente, com potencial aplicabilidade em alimentos, mas tem sido pouco estudada. É composta essencialmente de xilose, glicose e ácido glicurônico, formando um polissacarídeo ramificado (LIN, et al., 1994). Representa cerca de $5 \%$ a $6 \%$ da semente de chia e pode ser usada como fibra solúvel e dietética (REYESCAUDILLO, et al., 2008). Além disso, CAPITANI et al. (2012) afirmaram que as frações fibrosas da chia evidenciam uma grande capacidade de reter e absorver água, como um agente emulsionante e estabilizante de emulsões e o consumo dessa fibra dietética pode ser uma importante alternativa para melhorar a saúde humana.

Diante disso, o presente trabalho objetivou desenvolver sobremesas à base de soja, utilizando a mucilagem da chia na formulação em substituição ao agente espessante comumente utilizado (goma carragena), avaliando a influência desse constituinte sobre as características reológicas, físicas, químicas, cromáticas e sensoriais do produto.

\section{MATERIAL E MÉTODOS}

Processo de obtenção da mucilagem

As sementes de chia (Salvia hispanica L.) (Cacalia Comercial LTDA, Estância Velha, RS, Brasil) foram adquiridas em mercado local. Elas foram mergulhadas em água destilada em uma proporção de semente:água de 1:30, e deixadas sob agitação em agitador magnético (Fisatom, 752-A, SP, Brasil) por 2 horas em temperatura ambiente. A mucilagem formada foi então separada da semente através de filtração, com auxílio de uma bomba de vácuo e de uma peneira simples, e posteriormente centrifugada durante 15 minutos a 3000g. O sobrenadante (mucilagem) foi então congelado em ultrafreezer (LIOBRAS, UFR30, SP, Brasil) a $-70^{\circ} \mathrm{C}$, e após liofilizado (LIOTOP, L101, SP, Brasil) .

Preparo das sobremesas

Para o preparo das amostras foram utilizadas as seguintes matérias-primas: extrato de soja (Olvebra Industrial S/A, Eldorado do Sul, Brasil), carboximetilcelulose (CMC) (Hexus Food, Portão, Brasil) carragena (Cargill Alimentos, Minnesota, Estados Unidos), polidextrose (Danisco Brasil Ltda, Copenhague, Dinamarca), polpa de goiaba (Mais fruta, Antônio Prado, Brasil), amido modificado (Corn Products, Mogi-guaçu Brasil), sacarose (União, Limeira, Brasil) e suco em pó de goiaba (Kraft Foods, Curitiba, Brazil). A quantidade dos ingredientes (g $100 \mathrm{~g}^{-1}$ ) usados na formulação da sobremesa padrão foi: sacarose (9), polpa de goiaba (13), extrato hidrossolúvel de soja (5), amido modificado $(3,3)$, polidextrose (2), suco de goiaba em pó $(1,5)$, carragena $(0,3), \mathrm{CMC}(0,4)$ e água (quantum sufficit para 100g). Na formulação da amostra com chia, substituiu-se a carragena pela mucilagem dessa semente $(0,05 g)$. A formulação padrão foi obtida através de um planejamento fatorial completo rotacional $2^{3}$, previamente realizado, no qual avaliouse o efeito das seguintes variáveis: \% de extrato de soja (de 2,5 a 7,5), \% de polpa de goiaba (de 7 a 19) e \% de amido modificado (de 2,6 a 4,0) (dados não mostrados). As concentrações dos espessantes (gomas e mucilagem de chia) foram previamente testadas e avaliadas em função das características funcionais tecnológicas.

Para o preparo da amostra padrão, o extrato de soja foi hidratado em água a $40^{\circ} \mathrm{C}$ sob agitação mecânica, com impelidor tipo hélice naval (Fisaton, 713 D, Brasil). Logo após, os ingredientes secos foram adicionados, com exceção do amido, sob agitação durante $15 \mathrm{~min}$ a $40^{\circ} \mathrm{C}$. O amido gelatinizado e a polpa também foram adicionados e misturados durante 15min. Após a mistura dos ingredientes, as amostras foram colocadas em potes de vidro, previamente esterilizados em autoclave (Prismatec, CS, Brasil) $\left(120^{\circ} \mathrm{C}, 20\right.$ minutos), pasteurizadas e, em seguida, resfriadas à temperatura ambiente. Os produtos foram armazenados sob refrigeração $\left(7 \pm 1^{\circ} \mathrm{C}\right)$. Nas amostras elaboradas com chia, a mucilagem foi pré-hidratada durante 30min e colocada após a solubilização do extrato de soja em água. As etapas seguintes são exatamente iguais às mencionadas anteriormente.

\section{Análises físico-químicas}

O teor de sólidos solúveis e o $\mathrm{pH}$ foram determinados usando refratômetro analógico portátil (Carl Zeiss, 32-G, Estados Unidos) (AOAC, 2000a) e pHmetro (Tecnal, TEC-3MP, Brasil) (AOAC, 2000b), respectivamente. 
Análise de cor

As leituras colorimétricas foram feitas em um colorímetro (Konica Minolta Sensing Inc., CR 400, Japão). A partir do espectro de refletância da amostra, obtiveram-se os parâmetros colorimétricos $L^{*}, a^{*}$ e $b^{*}$ da escala CIELAB, conforme estabelecido pela Comission International de l'Eclairage (CIE). Os valores de $L^{*}$ variam de 0 (totalmente preta) a 100 (totalmente branca). A coordenada de cromaticidade $a^{*}$ varia de -80 (verde) a +100 (vermelho) e o $b^{*}$ varia de -50 (azul) a +70 (amarelo). A partir dos valores $a^{*} e b^{*}$, pode-se calcular a distância da coordenada $(a, b)$ em relação a sua origem $(0,0)$, o que é conhecido por cromaticidade ou Chroma $\left(C^{*}\right)$ e o ângulo de tonalidade $h$, que é medido em uma escala de 0 a $360^{\circ}$, determinando a localização correta da amostra em questão no espaço de cor.

As diferenças de cor foram determinadas pelo parâmetro $D E^{*}$, que foi calculado pela distância Euclidiana entre dois pontos em um espaço tridimensional, definidos pelos parâmetros $L^{*}, a^{*} e$ $b^{*}$. Matematicamente, o parâmetro colorimétrico $D E^{*}$ pode ser descrito pela Equação 1:

$$
\Delta E^{*}=\sqrt{\left(\Delta L^{*}\right)^{2}+\left(\Delta a^{*}\right)^{2}+\left(\Delta b^{*}\right)^{2}}
$$

\section{Análise reológica}

As análises reológicas foram realizadas em reômetro rotacional (Ares, TA Instruments, New Castle, USA), acoplado a um sensor de geometria placa-placa, e abertura (gap) de 1,5mm. O controle da temperatura foi realizado através de ar circulante. As curvas de escoamento foram feitas em duplicata a $25^{\circ} \mathrm{C}$ e a taxa de cisalhamento variou de 0 a $200 \mathrm{~s}^{-1}$. Os dados experimentais foram ajustados de acordo com o modelo reológico de Ostwald-de-Waelle (Lei da Potência, Equação 2), utilizando o software Statistica versão 10.0 (Statsoft Inc., Tulsa, Estados Unidos).

$$
\tau=K \dot{\gamma}^{n}
$$

em que: $\tau$, tensão de cisalhamento (N.m- ou Pa); $\gamma$, taxa de cisalhamento $\left(\mathrm{s}^{-1}\right) ; K$, índice de consistência (Pa.s); e $n$, índice de fluxo (adimensional).

\section{Determinação do perfil sensorial}

O perfil sensorial das amostras foi realizado por meio da Análise Descritiva Quantitativa (ADQ), descrita por STONE \& SIDEL (2004) com provadores treinados. Para tanto, as seguintes etapas foram executadas: Pré-seleção dos provadores: vinte membros do Instituto de Ciência e Tecnologia de Alimentos da Universidade Federal do Rio Grande do Sul (UFRGS) foram pré-selecionados com base na disponibilidade, interesse e na inexistência de alergia a algum componente da formulação. Os interessados em participar do painel sensorial preencheram um formulário informando dados pessoais e disponibilidade de horários para a realização das análises. Selecionaram-se 20 pessoas, 4 homens e 16 mulheres, com idades entre 18 e 68 anos. Testes classificatórios: a) Teste de diferenciação de sabores: foi realizado para verificar se os provadores eram capazes de distinguir os sabores básicos (doce, salgado, amargo e acido). b) Testes triangulares: foi realizada uma série de quatro repetições deste teste com duas sobremesas similares, porém com formulações diferentes. De vinte provadores recrutados voluntariamente, foram pré-selecionados quinze provadores, pela habilidade em discriminar a intensidade dos quatro sabores, assim como os que conseguiram no mínimo três respostas corretas nos testes triangulares. Desenvolvimento da terminologia descritiva: utilizou-se o Método de Rede de Kelly (MOSKOWITZ, 1983) para gerar os termos descritores que definem as similaridades e diferenças entre as amostras. Os provadores receberam dois pares de amostras, descrevendo as sensações observadas ao prová-las com relação à aparência, aroma, sabor e textura. Os julgadores discutiram os termos propostos e selecionaram consensualmente os termos a serem utilizados na ficha sensorial, utilizando escalas não estruturadas de $9 \mathrm{~cm}$, ancorada à esquerda com termos referentes ao mínimo e à direita pelo termo referente ao máximo para cada atributo, bem como os padrões de referência que foram utilizados para o treinamento (Tabela 1). Seleção e treinamento da equipe final: os candidatos foram então selecionados com base no poder de discriminação entre amostras, repetibilidade e concordância entre os julgadores, verificadas através de análise de variância de dois fatores (amostra e repetição) para cada provador, em relação a cada atributo. Avaliação das amostras: os provadores foram convocados a participar da avaliação das amostras. Na aplicação dos testes sensoriais, as amostras foram servidas resfriadas $\left(7^{\circ} \mathrm{C}\right)$ em copos plásticos codificados com números de três dígitos, de maneira aleatória, acompanhados de colheres e água mineral. Os testes foram realizados em cabines individuais no Laboratório de Análise Sensorial do Instituto de Ciência e Tecnologia de Alimentos da UFRGS. A análise microbiológica das amostras foi previamente realizada a fim de confirmar a segurança dos provadores.

\section{Análise estatística}

Os resultados da ADQ foram avaliados pela Análise de Variância (ANOVA) e teste de Tukey. Todas as análises estatísticas foram realizadas 
Tabela 1 - Definição dos termos descritivos e referências utilizadas como extremos de escala de intensidade na Análise Descritiva Quantitativa (ADQ) das sobremesas à base de soja para os atributos de aparência, aroma, sabor e textura.

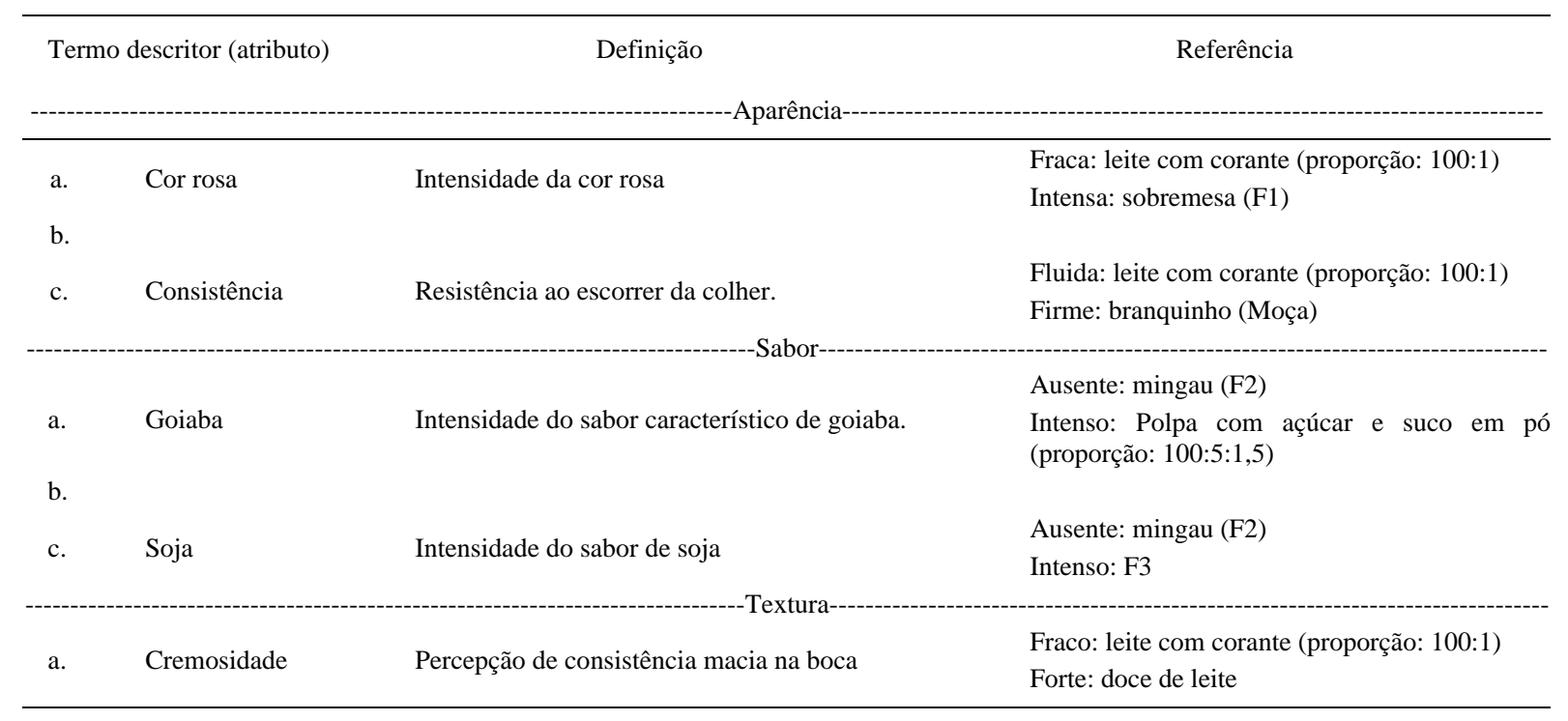

* F1: formulação padrão sem polpa de fruta e com $20 \mathrm{~g}$ de suco de goiaba em pó (g $100 \mathrm{~g}^{-1}$ ).

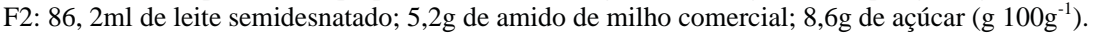

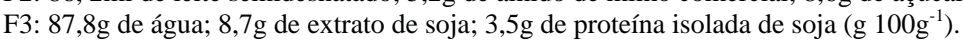

utilizando-se o software Statistica 10.0 (Statistica 10.0, Statsoft@, Tulsa, OK, USA) ao nível de 5\% de significância $(\mathrm{P}<0,05)$.

\section{RESULTADOS E DISCUSSÃO}

As amostras não diferiram significativamente quanto aos valores de $\mathrm{pH}$ e ${ }^{\circ} \mathrm{Brix}$. A amostra padrão

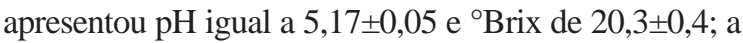
amostra com chia apresentou $\mathrm{pH}$ e conteúdo de sólidos

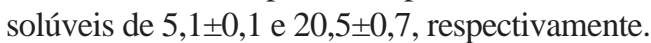

Através da colorimetria foi possível avaliar as alterações qualitativas na cor, promovidas pela incorporação da chia na sobremesa. A tabela 2 apresenta as coordenadas cromáticas de cada amostra, assim como a saturação da cor $\left(C^{*}\right)$, tonalidade $(h)$ e diferença de cor total $\left(\Delta \mathrm{E}^{*}\right)$.

Em relação à luminosidade, que varia do branco $\left(L^{*}=100\right)$ ao preto $\left(L^{*}=0\right)$, foi observado que a formulação com chia apresentou um valor significativamente superior, ou seja, mostrou maior luminosidade. O parâmetro cromático $b^{*}$, também diferiu significativamente entre as amostras; esses valores podem estar ligados à coloração mais amarelada, conferida pela carragena que foi inserida de maneira diferente da mucilagem (a qual foi previamente diluída, conferindo maior luminosidade à amostra). Sabendo que o $h$ entre $0^{\circ}$ e $90^{\circ}$ caracteriza a qualidade da cor de vermelho a amarelo (ALVES et al., 2008), pode-se afirmar que a amostra com chia apresentou uma coloração mais avermelhada.

Através das curvas de fluxo (dados não mostrados), foi identificado que as amostras apresentaram comportamento não newtoniano. Para ajustar aos dados das curvas de fluxo, foi utilizado o modelo de Ostwald-de-Waelle, estimando-se, para cada amostra, os valores dos parâmetros $K$ e $n$. Os valores dos parâmetros estimados para as amostras sem e com a adição de chia são apresentados nas eqs. 3 e 4, respectivamente. Em ambos os casos, o comportamento foi pseudoplástico $(0<\mathrm{n}<1)$, porém a adição de chia diminuiu significativamente a pseudoplasticidade da sobremesa. O coeficiente de consistência $(K)$, que também diferiu de forma

Tabela 2 - Parâmetros de cor das amostras de sobremesa à base de soja com adição de mucilagem de chia.

\begin{tabular}{lll}
\hline Parâmetros de cor & \multicolumn{1}{c}{ A } & B \\
\hline$L^{*}$ & $55.6 \pm 0.7^{\mathrm{b}}$ & $59.1 \pm 0.1^{\mathrm{a}}$ \\
$a^{*}$ & $4.9 \pm 0.2^{\mathrm{a}}$ & $4.6 \pm 0.2^{\mathrm{a}}$ \\
$b^{*}$ & $9.3 \pm 0.1^{\mathrm{a}}$ & $7.1 \pm 0.2^{\mathrm{b}}$ \\
$\mathrm{C}^{*}$ & $10.6 \pm 0.2^{\mathrm{a}}$ & $8.5 \pm 0.3^{\mathrm{b}}$ \\
$\mathrm{h}$ & $61.6 \pm 0.9^{\mathrm{a}}$ & $57.2 \pm 0.6^{\mathrm{b}}$ \\
$\Delta \mathrm{E}^{*}$ & & 4,12 \\
\hline
\end{tabular}

*Média de três repetições \pm desvio padrão. Letras distintas na linha diferem significativamente $(\mathrm{P}<0,05)$. 
significativa entre as amostras, foi superior nas amostras com carragena. Com esses parâmetros, é possível calcular a viscosidade dos produtos, visto que ela é um importante parâmetro de controle de qualidade durante um processo industrial. Dessa forma, pode-se afirmar que a amostra padrão, devido à sua maior pseudoplasticidade, está mais sujeita a mudanças em sua viscosidade durante sua produção.

$$
\begin{aligned}
\tau_{\mathrm{A}} & =(54,7 \pm 1,3) \gamma^{(0,29 \pm 0,03)} \\
\tau_{\mathrm{B}} & =(9,3 \pm 0,3) \gamma^{(0,47 \pm 0,02)}
\end{aligned}
$$

Os coeficientes de correlação $\left(\mathrm{R}^{2}\right)$ foram 0.993 e 0.986 para a amostra padrão e com chia, respectivamente, mostrando um bom ajuste dos dados. Os dados reológicos das curvas de fluxo de sobremesas analisadas por TÁRREGA \& COSTELL (2006), ARCIA et al. (2010), GONZÁLEZ-TOMÁS et al. (2008) e TÁRREGA \& COSTELL (2007) também foram adequados ao modelo de Ostwald-de-Waelle. $O$ valor de $K$ para a sobremesa com chia se aproximou do reportado por TÁRREGA \& COSTELL (2006), que estudaram sobremesas lácteas enriquecidas com inulina. Os valores encontrados por esses autores variaram de 0,4 a 12,9. Já os resultados de $n$ variaram de 0,3 a 0,6, valores similares aos encontrados neste trabalho. ARCIA et al. (2010) também estudaram sobremesas lácteas prebióticas e reportaram valores de $K$ entre 12,7 e 44 e de $n$ entre 0,34 e 0,44, resultados, também, próximos aos observados no presente trabalho. Sobremesas com baixo teor de gordura, à base de amido de tapioca e k-carragena, estudadas por GONZÁLEZ-TOMÁS et al.(2008), apresentaram valores de $K$ entre 2,19 e 19,12 e de $n$ entre 0,46 e 0,52 , valores mais similares aos reportados para a sobremesa com chia do que a amostra com carragena, fato que pode estar relacionado à baixa concentração de goma (0,01\%) utilizada por esses autores.

Pelo Método de Rede de Kelly, descrito anteriormente, obtiveram-se as definições dos atributos descritivos para as amostras de sobremesa, conforme a tabela 1.

A tabela 3 apresenta as médias de cada atributo avaliado para cada amostra. As amostras não diferiram significativamente entre si em nível de 5\% em relação aos atributos cor rosa, sabor de soja, sabor de goiaba e cremosidade. Observou-se que a adição de mucilagem de chia diminuiu apenas a consistência aparente, demonstrando que esta substituição é válida, tendo em vista os benefícios proporcionados por ela.

TORREZAN et al. (2004), ao verificarem o perfil sensorial de bebidas comerciais com soja sabor laranja, obtiveram escores de 2,10 a 4,50 em uma escala de 9 pontos para o atributo sabor soja,
Tabela 3 - Média dos atributos selecionados pela equipe treinada para as sobremesas com soja.

\begin{tabular}{lcc}
\hline \multirow{2}{*}{ Atributo } & ------------Média da equipe sensorial------------- \\
& Formulação padrão - A Formulação com chia -B \\
\hline Cor rosa & $2,77 \pm 1,20^{\mathrm{a}}$ & $2,08 \pm 1,49^{\mathrm{a}}$ \\
Consistência & $5,83 \pm 1,27^{\mathrm{a}}$ & $3,30 \pm 1,86^{\mathrm{b}}$ \\
Sabor soja & $1,15 \pm 1,95^{\mathrm{a}}$ & $0,91 \pm 1,17^{\mathrm{a}}$ \\
Sabor goiaba & $4,84 \pm 2,54^{\mathrm{a}}$ & $5,21 \pm 2,16^{\mathrm{a}}$ \\
Cremosidade & $6,81 \pm 0,55^{\mathrm{a}}$ & $6,07 \pm 0,96^{\mathrm{a}}$ \\
\hline
\end{tabular}

* Média de três repetições \pm desvio padrão. Letras distintas na linha diferem significativamente $(\mathrm{P}<0,05)$.

valores superiores aos reportados neste trabalho. Já SANTANA et al. (2006) determinaram o perfil sensorial de iogurte light comercial sabor pêssego e verificaram que a proteína de soja contida na versão do produto "adicionado de proteína de soja” não interferiu na aceitação do consumidor.

Diferente do presente trabalho, que utilizou polpa de fruta para mascarar o sabor de soja, TORRES-PENARANDA \& REITMEIER (2001) utilizaram diferentes linhagens do grão (livres de lipooxigenase) com esse mesmo propósito e fizeram análise descritiva quantitativa de leite de soja. Tal como esperado, os autores verificaram que a intensidade do sabor de soja foi menor nos leites de soja a partir de grãos livres de lipooxigenase do que para o leite com grão sem modificação. TÁRREGA \& COSTELL (2007) avaliaram o perfil sensorial de sobremesas lácteas comerciais sabor baunilha e também detectaram diferenças significativas na cremosidade das amostras, fato relacionado à formulação dos produtos, que diferiu quanto aos componentes (amido, carragena, goma guar, goma xantana etc.) e quantidade destes.

\section{CONCLUSÃO}

Este estudo mostrou que a substituição da carragena em sobremesas à base de soja pela mucilagem de chia não proporcionou grandes modificações nas características, tais como $\mathrm{pH}$, ${ }^{\circ}$ Brix, coordenada cromática $a^{*}$ e nos atributos sensoriais cor rosa, sabor de goiaba, sabor de soja e cremosidade. A referida substituição teve efeito significativo sobre o comportamento reológico do produto, tornando-o menos viscoso, porém também menos pseudoplástico. Essas mudanças reológicas foram percebidas apenas na consistência visual da amostra. 


\section{AGRADECIMENTOS}

Os autores agradecem ao Conselho Nacional de Desenvolvimento Científico e Tecnológico (CNPQ) pelo apoio financeiro.

\section{COMITÊ DE ÉTICA}

Todos os provadores receberam e assinaram o Termo de consentimento esclarecido aprovado pelo Comitê de ética da UFRGS (protocolo Número 91.497).

\section{REFERÊNCIAS}

ALM, L. Lactose intolerance. In: ROGINSKY, H. et al. (Eds.) Encyclopedia of dairy sciences. London: Academic, 2002. p.1533-1553.

ALVES, C.C.O. et al. Estabilidade da microestrutura e do teor de carotenóides de pós obtidos da polpa de pequi (Caryocar brasiliensi camb.) liofilizada. Ciência e Tecnologia de Alimentos, v.28, n.4, p.830-839, 2008. Disponível em: <http://www.scielo.br/ scielo.php?pid=S0101-20612008000400011\&script=sci_arttext $>$. Acesso em: 20 jan.2013.doi:10.1590/S0101-20612008000400011.

AOAC. Official Method 932.12. Gaithesburg, 2000a. 1200p.

AOAC. Official Method 981.12. Gaithesburg, 2000b. 1200p.

ARCIA, P.L. et al. Thickness suitability of prebiotic dairy desserts: Relationship with rheological properties.Food Research International, v.43, n.10, p.2409-2416, 2010 Disponível em: <http://www.sciencedirect.com/science/article/pii/ S0963996910003212>. Acesso em: 20 jan. 2013.doi: 10.1016/j. foodres.2010.09.013.

CAPITANI, M.I. et al. Physicochemical and functional characterization of by-products from chia (salvia hispanica l.) seeds from argentina. Food Science and Technology, v.45, p.94-102, 2012. Disponível em: <http://www.sciencedirect.com/ science/article/pii/S0023643811002131>. Acesso em: 22 jan. 2013. doi: 10.1016/j.lwt.2011.07.012.

FRIEDMAN, M.; BRANDON, D.L. Review: nutritional and health benefits of soy proteins. Journal of Agricultural and Food Chemistry, v.49, p.1069-1086, 2001. Disponível em: <http://www.ncbi.nlm.nih.gov/pubmed/11312815>.Acesso em: 22 jan.2013.doi:10.1021/jf0009246.

GONZÁLEZ-TOMÁS, L. et al. Rheology, flavour release and perception of low-fat dairy desserts. International Dairy Journal, v.18, n.8, p.858-866. 2008. Disponível em: <http:// www.sciencedirect.com/science/article/pii/S0958694607001872>. Acesso em: 25 jan. 2013. doi: 10.1016/j.idairyj.2007.09.010.

LIN, K.Y. et al. Structure of chia polysaccharide exudate. CarbohydratePolymers, v.23,p.13-18,1994.Disponívelem: <http:// www.sciencedirect.com/science/article/pii/014486179490085X>. Acesso em: 18 jan. 2013. doi: 10.1016/0144-8617(94)90085-X.
MOSKOWITZ, H.R. Product testing and sensory evaluation of foods. Westport: Food e Nutrition, 1983. 605p.

MUÑOZ, L.A. et al. Characterization and microstructure of films made from mucilage of salvia hispanica and whey protein concentrate. Journal of Food Engineering, v.111, p.511-518. 2012. Disponível em: <http://www.sciencedirect.com/science/ article/pii/S0260877412001112>. Acesso em: 10 fev. 2013. doi: 10.1016/j.jfoodeng.2012.02.031.

PEIRETTI, P.G.; GAI, F. Fatty acid and nutritive quality of chia (Salvia hispanica 1.) seeds and plant during growth. Animal Feed Science Technology, v.148, p.267-275, 2009. Disponível em: <http:// www.sciencedirect.com/science/article/pii/S0377840108001405>. Acesso em: 07 fev. 2013. doi: 10.1016/j.anifeedsci.2008.04.006.

REYES-CAUDILLO, E. et al. Dietary fibre content and antioxidant activity of phenolic compounds present in mexican chia (Salvia hiINspanica l.) seeds. Food Chemistry, v.107, p.656-663, 2008. Disponível em: <http://www.sciencedirect.com/science/article/pii/ S0308814607008709>. Acesso em: 23 jan. 2013. doi: 10.1016/j. foodchem.2007.08.062.

SANTANA, L.R.R. et al. Perfil sensorial de iogurte light, sabor pêsssego. Ciência e Tecnologia de Alimentos, v.26, n.3, p.619625, 2006. Disponível em: <http://www.scielo.br/scielo.php?pid =s010120612006000300021\&script=sci_arttext $>$. Acesso em: 08 fev. 2013. doi: 10.1590/S0101-20612006000300021.

SOYFOODS: sales and trends. Disponível em: <http://www. soyfoods.org/products/sales-and-trends>. 2010. Acesso em: 06 fev. 2013.

STONE, H.; SIDEL, J.L. Descriptive analysis. Sensory evaluation practices. London: Academic, 2004. 311p.

TÁRREGA, A.; COSTELL, E. Effect of inulin addition on rheological and sensory properties of fat-free starch-based dairy desserts. International Dairy Journal, v.16, n.9, p.1104-1112, 2006. Disponível em:<http://www.sciencedirect.com/science/ article/pii/S0958694605001810>. Acesso em: 24 jan. 2013. doi: 10.1016/j.idairyj.2005.09.002.

TÁRREGA, A.; COSTELL, E. Colour and consistency of semisolid dairy desserts: Instrumental and sensory measurements. Journal of Food Engineering, v.78, n.2, p.655-661,2007. Disponível em: <http://www.sciencedirect.com/science/article/pii/ S026087740500751X>. Acesso em: 04 fev. 2013. doi: 0.1016/j. jfoodeng.2005.11.003.

TORRES-PENARANDA, A.V.; REITMEIER, C.A. Sensory descriptive analysis of soymilk. Journal of Food Science, v.66, n.2, p.352-356, 2001.Disponível em: <http://onlinelibrary.wiley. com/doi/10.1111/j.1365-2621.2001.tb11345.x/pdf>. Acesso em: 19 fev. 2013. doi: 10.1111/j.1365-2621.2001.tb11345.x.

TORREZAN, R.E.A. et al. Avaliação do perfil sensorial de alimento com soja sabor laranja. Boletim CEPPA, v.22, n.2, p.199-216, 2004. Disponível em: <http://ojs.c3sl.ufpr.br/ojs2/ index.php/alimentos/article/viewArticle/1190>. Acesso em: 29 jan. 2013. ISSN:19839774. 